\title{
Comparación Del Estrato Arbóreo En Fragmentos De Bosques Urbanos De Margarita, Espinar Y Davis, Provincia De Colón, República De Panamá
}

Comparison Of Tree Stratum In Urban Forest Fragments Of Margarita, Espinar And Davis, Province Of Colón, Republic Of Panama.

\author{
Ana Victoria Zúñiga ${ }^{1}$, Francisco Farnum Castro². y Vielka Murillo Godoy ${ }^{3}$
}

\author{
Universidad de Panamá, Centro Regional Universitario de Colón, Panamá, Panamá \\ 1victoriadegracia@hotmail.com. \\ 2 frank0523@hotmail.com [https://orcid.org/0000-0002-5879-2296] \\ 3vielkam@gmail.com [https://orcid.org/0000-0002-6284-4466]
}

\section{Resumen}

Un conjunto de fragmentos de bosques urbanos muy valiosos son los que se encuentran en los poblados de Margarita, Espinar y Davis, que están cercanos o en la periferia de las instalaciones civiles, residenciales y militares de las antiguas bases militares estadounidenses en la Provincia de Colón e incluidos dentro de las áreas de operación del Canal de Panamá. Los bosques urbanos por estar expuestos enfrentan muchas amenazas planteadas por la urbanización no regulada, la falta de gestión e inversión en el mantenimiento de los mismos, por lo tanto el objetivo de esta investigación es el de conocer la flora existente en las áreas de estudio para conocer los riesgos y amenazas que enfrenta la misma. Esta investigación se realizó de agosto a octubre de 2017 y se realizaron evaluaciones visuales a los árboles inventariados, se les asignó un valor, según el nivel de riesgo determinado en la inspección realizada, lo que permitió identificar las especies que presentaron prioridad para su futura valoración de riesgo. Se registró la presencia de 22 familias, con 42 géneros y 63 especies. Las familias mejor representadas fueron: Arecaceae y Lythraceae. Los resultados de esta investigación señalan que los árboles de las áreas estudiadas presentan buen estado en su mayoría, y es importante el mantenimiento de los mismos para que en el futuro sigan brindando los beneficios que hasta ahora proporcionan como pulmones de las áreas estudiadas.

Palabras Clave: Estrato Arbóreo, Bosques Urbanos, Fragmentos Boscosos, Flora Urbana, Amenazas. 


\begin{abstract}
A set of very valuable urban forest fragments are those found in the towns of Margarita, Espinar and Davis, which are close to or on the periphery of the civil, residential and military installations of the old US military bases in the Province of Colón and included within the areas of operation of the Panama Canal. Due to being exposed, urban forests face many threats posed by unregulated urbanization, the lack of management and investment in their maintenance, therefore the objective of this research is to know the flora existing in the study areas to know the risks and threats it faces. This research was carried out from August to October 2017 and visual evaluations were made to the inventoried trees, a value was assigned, according to the level of risk determined in the inspection carried out, which allowed identifying the species that presented priority for their future valuation risky. The presence of 22 families was recorded, with 42 genera and 63 species. The best represented families were: Arecaceae and Lythraceae. The results of this research indicate that the trees in the areas studied are mostly in good condition, and it is important to maintain them so that in the future they continue to provide the benefits that they provide up to now as lungs in the areas studied.
\end{abstract}

Keywords: Tree Stratum, Urban Forests, Forest Fragments, Urban Flora, Threats

\title{
Introducción
}

Hasta hace poco tiempo la perspectiva sobre los bosques urbanos se había restringido para aspectos recreativos y de esparcimiento, sin otorgarle la importancia a los servicios ambientales que le brindan al entorno. Sin embargo, este punto de vista ha cambiado en los últimos años, se ha generado una creciente consciencia de que estas áreas proporcionan múltiples beneficios, más allá de los estéticos y recreativos, mejorando la calidad de vida de sus habitantes (Farnum y Murillo, 2014).

El concepto "bosque urbano o área verde urbana" hace referencia al conjunto de recursos naturales: agua, suelo, clima, paisaje, plantas y organismos asociados, que se desarrollan relacionados con grupos humanos, cerca de edificios, en jardines públicos y privados, en parques, en lotes baldíos, cementerios y otros; este concepto amplía la perspectiva del importante y diverso papel que poseen estas áreas para disminuir los impactos negativos de la urbanización sobre los 
ecosistemas locales y el mejoramiento de la calidad ambiental de las ciudades las cuales constituyen actualmente el hábitat humano dominante en el planeta (Farnum y Murillo, 2014).

Actualmente el reemplazo indiscriminado de los bosques por estructuras de cemento, asfalto, metal y vidrio incrementa la temperatura y reduce la calidad de vida en las ciudades. Estos aumentos de la temperatura en las localidades urbanas se acentúan con el calentamiento global, el cual es inducido principalmente por la acumulación en la atmósfera de residuos de la quema de combustibles fósiles, tales como los óxidos de nitrógeno y el dióxido de carbono (Ibrahim et al., 2007). Todo es esto es contrarrestado con el efecto refrigerante de los bosques, los cuales además son sumideros del carbono que asimilan por fotosíntesis y fijan en sus estructuras de celulosa durante los largos períodos de vida de los árboles, quienes además liberan una mejor calidad de oxígeno que ayuda el microclima del lugar donde se encuentran, lo que tiene un efecto directo en el bienestar humano (sombra) y sobre el presupuesto para enfriar edificios (Balvanera, 2012).

Debido a su capacidad para retener el suelo y facilitar la absorción del agua lluvia, los bosques urbanos también funcionan como un componente del sistema de control de inundaciones. Al mismo tiempo, su papel significativo en la captura de agua de lluvia y su infiltración al subsuelo, propicia la recarga de los mantos acuíferos, favoreciendo con ello las reservas de agua para las ciudades.

La existencia del arbolado urbano constituye el hábitat de un gran número de poblaciones de aves y otros animales que encuentran refugio dentro del entorno urbano, particularmente, aquellos que habitaban el ecosistema original antes de extenderse la mancha urbana y gracias a estas zonas arboladas se puede sobrellevar el impacto de la presencia humana.

Estudios realizados en México sobre los bosques urbanos reportan que los bosques urbanos tienen la capacidad de disminuir la temperatura entre $2^{\circ}$ y $4^{\circ} \mathrm{C}$ en promedio, es el caso del Bosque Los Colomos, al noreste de Guadalajara, en el que se ha encontrado una variación hasta de 8 grados dentro de la zona boscosa, con relación a las plazas comerciales cercanas (Díaz y Ballesteros, 2012) . Otros estudios interesantes son los del Parque Nacional de Tijuca, Río de Janeiro, Brasil, considerado este como el parque urbano más grande del mundo. Desde comienzos del siglo XIX el parque y su bosque se han visto afectados por distintos episodios de degradación ambiental como consecuencia de la deforestación, la proliferación de especies exóticas y la presión de la expansión urbana. Las iniciativas de restauración emprendidas incluyeron la reforestación del parque, principalmente mediante la utilización de árboles nativos de la región. Otra medida fue crear infraestructuras recreativas dentro 
del mismo, lo que contribuyó a sensibilizar a la opinión pública local acerca de la importancia de proteger los bosques urbanos (Trzyna, 2014).

Desde 1999, el parque ha sido gestionado conjuntamente por la ciudad de Río de Janeiro y el Ministerio de Medio Ambiente. En la actualidad constituye un entorno natural excepcional para sus 2,5 millones de visitantes anuales, y la restauración del bosque atlántico ha permitido convertirlo en un santuario para la diversidad y el endemismo de las especies. Estos valiosos servicios contribuyeron a que, en 2012, el Parque Nacional de Tijuca recibiese el título de paisaje cultural patrimonio de la humanidad. (Trzyna, 2014.).

Por otro lado, en el año 2006, un estudio realizado por la FAO en Bangladesh, analizó la contribución de los bosques urbanos a los medios de vida para las poblaciones pobres de la ciudad. Se encontró que la silvicultura urbana puede aumentar la calidad de vida de la gente pobre, por ejemplo, por medio del empleo en los viveros y otras industrias forestales. La mayoría de los pobres de Dhaka carecen de acceso a los servicios de la ciudad, tales como el suministro de electricidad, agua potable y otros beneficios. La silvicultura urbana puede ayudar a salvar esta brecha, por ejemplo, produciendo combustible leñoso y ayudando a purificar el agua. La mayoría de los pobres de Dhaka trabaja al aire libre donde sufren condiciones ambientales peligrosas producidas por la contaminación del aire; los bosques urbanos pueden contribuir a mejorar estas condiciones. Los árboles ofrecen refugio y seguridad a los indigentes, además de lugares de recreo para los niños y niñas en la calle. En fin, la participación de los pobres en los procesos de toma de decisiones sobre los recursos forestales urbanos puede ofrecer un determinado nivel de empoderamiento. (Uddin, 2006; Sohel, Mukul y Burkhard ,2014).

En los últimos años se ha reconocido la importancia de los bosques urbanos en la sostenibilidad ambiental del planeta tierra. Se ha exaltado la contribución a nivel mundial para lograr el cumplimiento de los ODS propuestos en la Agenda 2030 (ONU, 2015) como un compromiso asumido por países para hacer frente a los desafíos del desarrollo y agotamiento de los recursos naturales.

No cabe duda que los beneficios y las desventajas del bosque urbano están influenciados por su planificación y manejo, como la ubicación y configuración de las plantaciones de árboles, la densidad de los rodales, la selección de especies y más.

Entre los bosques urbanos de Panamá está un fragmento valioso de bosques en el área de Margarita, Espinar y Davis en la provincia de Colón, bosques ricos en biodiversidad, cercanos o en la periferia de las instalaciones civiles, residenciales y militares de las bases militares estadounidenses en Panamá, bosques que a su vez están incluidos, dentro de las áreas de operación del Canal de Panamá, cercanos a la ciudad de Panamá y Colón. Estos bosques no escapan de las amenazas planteadas por la urbanización no regulada y la falta de inversiones y 
gestiones organizadas para su conservación. A pesar de que estos bosques brindan una amplia gama de bienes y servicios ecosistémicos y contribuyen enormemente a los medios de vida y a la calidad de vida de los habitantes de las zonas urbanas de Margarita, Davis y Espinar carecen de reglamentación por parte del estado, por consiguiente, no tienen legislación, ni normativa parecida a la de los parques nacionales y áreas protegidas del país, por lo que este arbolado es muy vulnerable y las amenazas de estos son mayores y más frecuentes.

Al no existir normativas que regulen los bosques urbanos se hace necesario y urgente hacer estudios como este, cuyo objetivo es conocer la flora existente y así comparar el estado ambiental y riesgo de los árboles presentes en las comunidades de Margarita, Espinar, Davis, mediante un estudio florístico, ya que conociendo las bondades de los bosques urbanos es importante tener en cuenta, que los árboles urbanos conllevan un riesgo o potencial de fallo que puede poner a las personas en peligro, dañar sus propiedades o ambos, aspecto que ha ido creciendo en los habitantes (Koeser, Hauer, Miesbauer y Peterson, 2016). Las situaciones de riesgo del árbol se pueden controlar parcialmente, pero no se puede eliminar completamente sin suprimir cada árbol. Por ello, el desafío está en desarrollar una adecuada gestión con el fin de asegurar un nivel de riesgo aceptable para sus gestores, compañías de seguros y la sociedad en general (Tomao et al., 2015). Por ejemplo, un árbol longevo sin mantenimiento tiene más probabilidad de fallar, por lo que es importante identificar dónde un árbol se ha convertido en un riesgo inaceptable a consecuencia de su deterioro natural, por estrés hídrico o térmico (O’Brien, 2003).

Este estudio aportará las bases para que se elaboren los planes de manejo y conservación de los fragmentos boscosos de Margarita, Davis y Espinar, pues sólo conociendo qué árboles se reportan en estas áreas y su condición de riesgo y usos, se puede valorar con mayor amplitud la riqueza vegetal existente, la que demanda manejo y toma de decisiones para la conservación de las mismas, de manera que sean transferidas como legado a las futuras generaciones.

Cada vez hay más pruebas de que las instituciones gubernamentales ya no son los únicos actores importantes en los procesos de toma de decisiones, y un ingrediente clave de la gestión sostenible de los bosques urbanos y periurbanos es, por lo tanto, la gobernanza inclusiva (Lawrence et al., 2013). Hay una creciente tendencia a reconocer a los actores de la sociedad civil como socios importantes en los análisis de políticas y en la promoción de los potenciales beneficios de los bosques urbanos y periurbanos. Todo esto nos lleva a entender que los habitantes o pobladores cercanos a los bosques urbanos están demostrando ya su compromiso con un futuro más sostenible. 


\section{Metodología}

Este estudio es de tipo descriptivo, transversal. La investigación se realizó de agosto a octubre de 2017, en los fragmentos boscosos de Margarita, José Domingo Espinar (Espinar) y José Dominador Bazán (Davis), localizados en el corregimiento de Cristóbal, (9¹9 '53" N, 7954'27" O) provincia de Colón, Republica de Panamá (ver Figuras 1, 2 y 3).Es un bosque urbano húmedo Tropical con altura de 8 m.s.n.m., temperatura de $26^{\circ} \mathrm{C}$, precipitación de 3,400 $\mathrm{mm}$ y humedad relativa de $85 \%$.

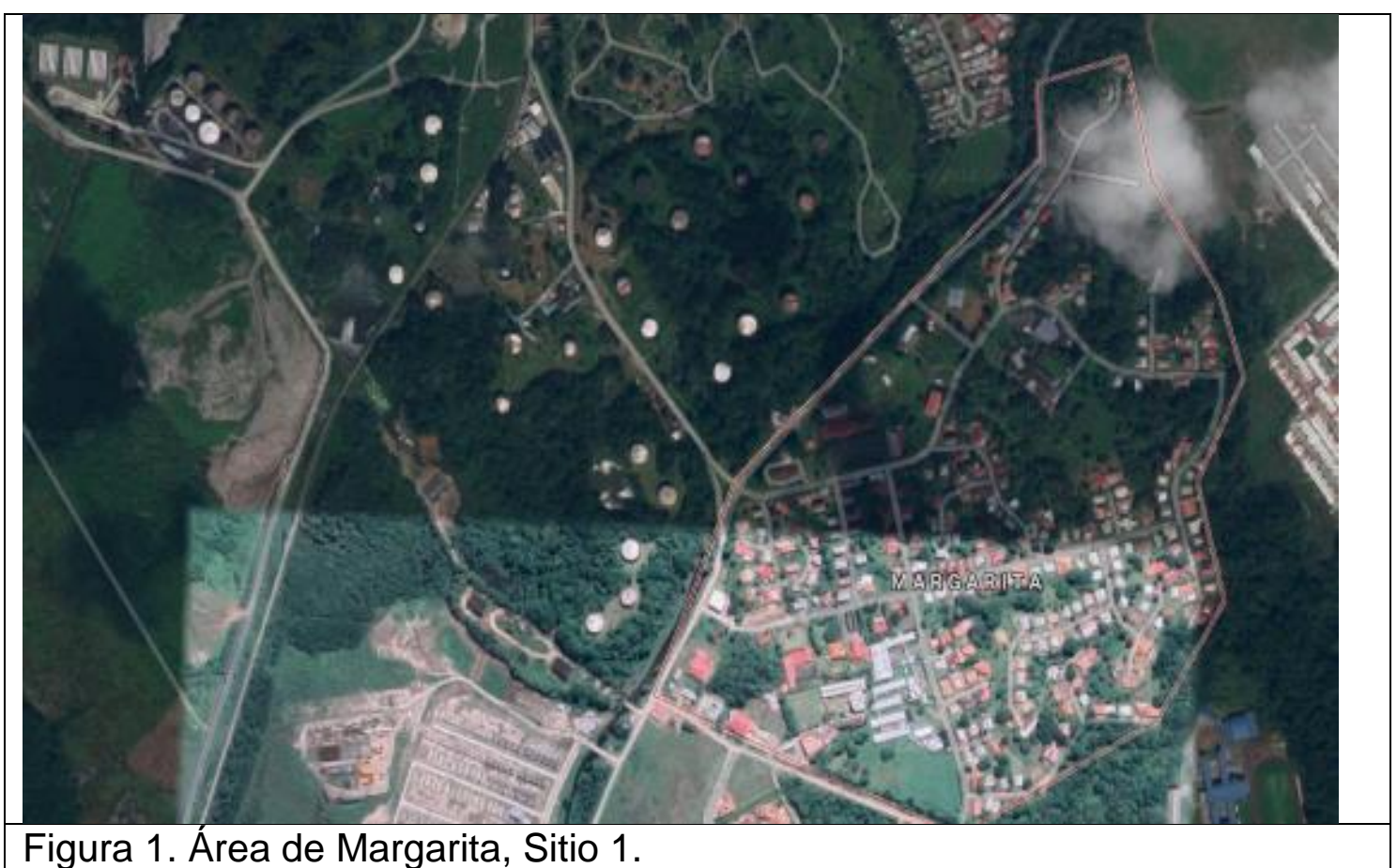



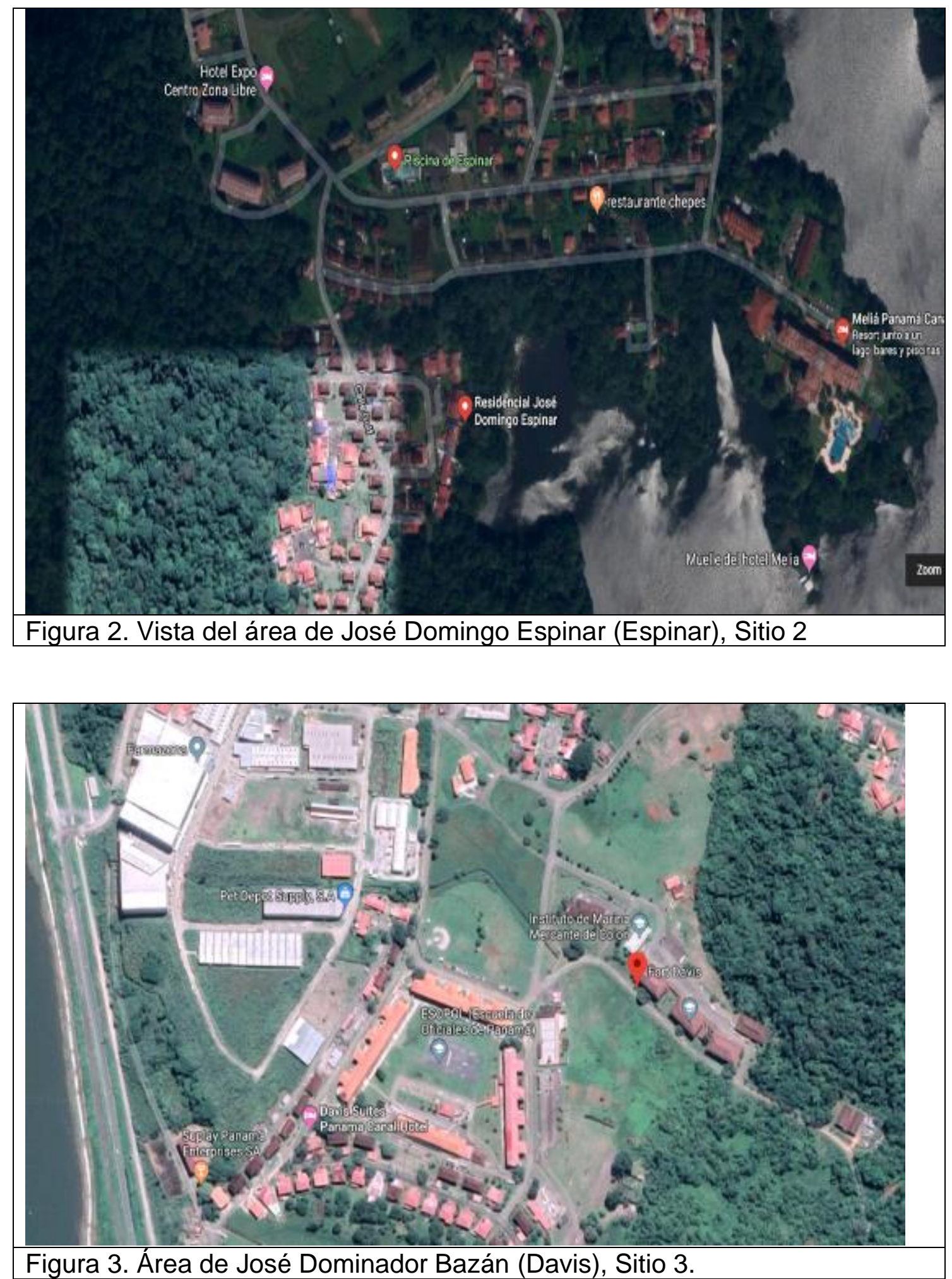

\section{Inventario de árboles}

Se empleó un muestreo aleatorio estratificado donde se dividió el área en tres estratos (los tres ecosistemas en estudio). Para las observaciones en campo, se realizaron visitas a cada sitio, donde se recorrieron los principales senderos de ellos. 
Se marcaron transectos lineales, realizándose anotaciones sobre las especies arbóreas presentes de:

1. Abundancia de cada individuo

2. Habito y simetría

3. Características etnobotánicas proporcionadas por la comunidad.

\section{Reconocimiento de las especies arbóreas}

Para el reconocimiento de los árboles, los mismos fueron marcados con una cinta de color amarillo, debidamente rotulados secuencialmente. Se tomaron muestras fértiles, fotografías del hábito completo, hojas y cualquier característica propia para cada hábito, con el fin de que estas fueran estudiadas y debidamente identificadas taxonómicamente.

La identificación final de las muestras botánicas se realizó con el apoyo de los especialistas del Centro Regional Universitario de Colón y la colección del Herbario de la Universidad de Panamá (PMA), las muestras fueron identificadas hasta la categoría taxonómica de especie.

Los datos recaudados se procesaron usando el programa Microsoft Excel versión 2013, los resultados se presentaron en tablas y gráficos.

\section{Estado de salud de los árboles}

Se realizaron evaluaciones visuales a los árboles inventariados y se les asignó un valor, según el nivel de riesgo determinado en la inspección realizada (ver Tabla 1), lo que permitió identificar las especies que presentaron prioridad para su futura valoración de riesgo. Tomando como partida el valor de riesgo arrojado y teniendo en cuenta la edad, el tamaño, y las especies existentes, se elaboró una lista en la que se concretó qué arbolado necesitaba con mayor urgencia la atención preventiva individualizada (ver Tabla 2). Esto se analizó mediante el nivel de riesgo acumulado que se calcula sumando el valor que hay en cada una de las columnas de la Tabla 1.

Tabla 1. Valoración del estado de riesgo de los árboles en bosques urbanos.

1: Riesgo $\quad$ Especie arbórea que no presenta raíces expuestas en demasiadas cavidades, grietas o desgajes presentes en un $40 \%$ en tronco y ramas, anclaje débil y, por tanto, que muestre una estructura natural bien distribuida y estable, así como follaje vigoroso. 


\begin{tabular}{|l|l|}
\hline $\begin{array}{l}\text { 2: Riesgo } \\
\text { medio y } \\
\text { apreciable }\end{array}$ & $\begin{array}{l}\text { Especie que puede tener más de una de las condiciones mencionadas en el párrafo anterior } \\
\text { siempre y cuando, estas no pongan en peligro la salud de los demás. Se caracterizan por tener } \\
\text { daños menores de insectos o enfermedades, por problemas fisiológicos reflejados en la calidad } \\
\text { del follaje, etc. Pueden mostrar daños mecánicos menores, plaga o enfermedades, pero la } \\
\text { muerte no es inminente. }\end{array}$ \\
\hline 3: Existe un & $\begin{array}{l}\text { Árbol que presenta anclaje débil, inclinación del tronco hacia una infraestructura, mayor a los } \\
45^{\circ} \text { o alguna fitopatología en la madera (de más del 51\% en tronco ramas y raíces), estén cerca } \\
\text { de cables eléctricos, o que se encuentren moribundo. También se consideran a todos aquellos } \\
\text { individuos que representen un riesgo inmediato a los habitantes o daños a la propiedad de las } \\
\text { riesgo } \\
\text { inminente o alto }\end{array}$ \\
\hline \multicolumn{2}{|l|}{ Fuente. Elaboración propia. (2019). } \\
\hline
\end{tabular}

Tabla 2. Categorías de riesgo o amenaza, de poda o tala para los árboles urbanos

\begin{tabular}{|c|c|c|c|c|c|c|c|c|c|c|c|}
\hline $\begin{array}{l}\text { Nombre } \\
\text { científico }\end{array}$ & $\begin{array}{l}\text { Tiene } \\
\text { alto } \\
\text { valor } \\
\text { de } \\
\text { uso }\end{array}$ & $\begin{array}{l}\text { Se } \\
\text { encuentra } \\
\text { próximo a } \\
\text { la vía o } \\
\text { acera }\end{array}$ & $\begin{array}{l}\text { Se } \\
\text { encuentra } \\
\text { próximo a } \\
\text { residencias }\end{array}$ & $\begin{array}{l}\text { Tiene } \\
\text { avanzada } \\
\text { edad }\end{array}$ & $\begin{array}{l}\text { Muestra } \\
\text { signos de } \\
\text { enfermedad }\end{array}$ & $\begin{array}{l}\text { Su tipo de } \\
\text { crecimiento } \\
\text { actual es } \\
\text { una } \\
\text { amenaza }\end{array}$ & $\begin{array}{l}\text { Obstaculiza } \\
\text { el paisaje o } \\
\text { alguna obra }\end{array}$ & $\begin{array}{l}\text { Produce } \\
\text { abundante } \\
\text { material } \\
\text { orgánico }\end{array}$ & $\begin{array}{l}\text { Atrae } \\
\text { insectos } \\
u \text { otros } \\
\text { animales }\end{array}$ & $\begin{array}{l}\text { Tiene un } \\
\text { historial } \\
\text { de daños } \\
\text { a la } \\
\text { propiedad }\end{array}$ & $\begin{array}{l}\text { Nivel de } \\
\text { riesgo } \\
\text { acumulado }\end{array}$ \\
\hline & & & & & & & & & & & \\
\hline
\end{tabular}

\section{Valoración etnobotánica}

En el área de estudio se realizaron conversaciones con los habitantes de mayor edad, se les indagó mediante una encuesta de repuesta cerrada sobre el conocimiento que tenían de las plantas al mencionarle los distintos usos: alimento, medicinal, maderable, ornamental, artesanal, industrial y tradiciones. Con el índice de valor de uso se evaluó el valor de uso de las especies.

\section{Resultados Y Discusión}

\section{A. Inventario Florístico}

La diversidad de especies de este estudio florístico de las comunidades de Margarita, Espinar y Davis reportó un total de 63 especies de árboles y arbustos representadas en 467 individuos, distribuidos en 22 familias. Las familias más representadas, según la cantidad de individuos, fueron: Arecaceae, con la especie Roystonea regia (Palma real cubana) que fue la especie más abundante del estudio representando casi un $30 \%$ del total de las plantas encontradas; otra especie destacada de esta familia fue Cocos nucifera (Palma de coco). 
Otras familias que tuvieron presencia fueron la Lythraceae con la especie Lagerstroemia speciosa (Reina de las flores o Crespón); Meliaceae con la especies Swietenia macrophylla (Caoba) y Cedrela odorata (Cedro amargo), Anacardiaceae con Mangifera indica (Mango), Malvaceae con Apeiba tibourbou (Peine de mico ) y Simaroubaceae con Simarouba amara (aceituno).

Además se encontraron otras 20 familias con individuos solitarios, con dos, tres, seis o más de 10 individuos, entre ellas: Boraginaceae, Cochlospermaceae Euphorbiaceae, Rubiaceae, Sapotaceae, Lauraceae, entre otras (ver Tabla 3 y Figura 4).

Es importante destacar que las especies más destacadas Roystonea regia, Cocos nucifera y Lagerstroemia speciosa son especies introducidas cultivadas y obedecen a una distribución planificada artificial, antrópica de paisajismo. A partir de esto es posible tener en cuenta que estas especies fueron seleccionadas posiblemente por su adaptabilidad, crecimiento rápido, por razones estéticas o de costos.

Tabla 3. Familias más representadas según el número de individuos en las comunidades de Margarita, Espinar y Davis.

\begin{tabular}{|l|r|}
\hline \multicolumn{1}{|c|}{ Familia } & Número de individuos \\
\hline Arecaceae & 161 \\
\hline Lythraceae & 41 \\
\hline Meliaceae & 26 \\
\hline Anacardiaceae & 20 \\
\hline Lecythidaceae & 22 \\
\hline Bignoniaceae & 21 \\
\hline Malvaceae & 19 \\
\hline Poaceae & 14 \\
\hline Simaroubaceae & 13 \\
\hline
\end{tabular}

Fuente. Elaboración propia. (2019) 


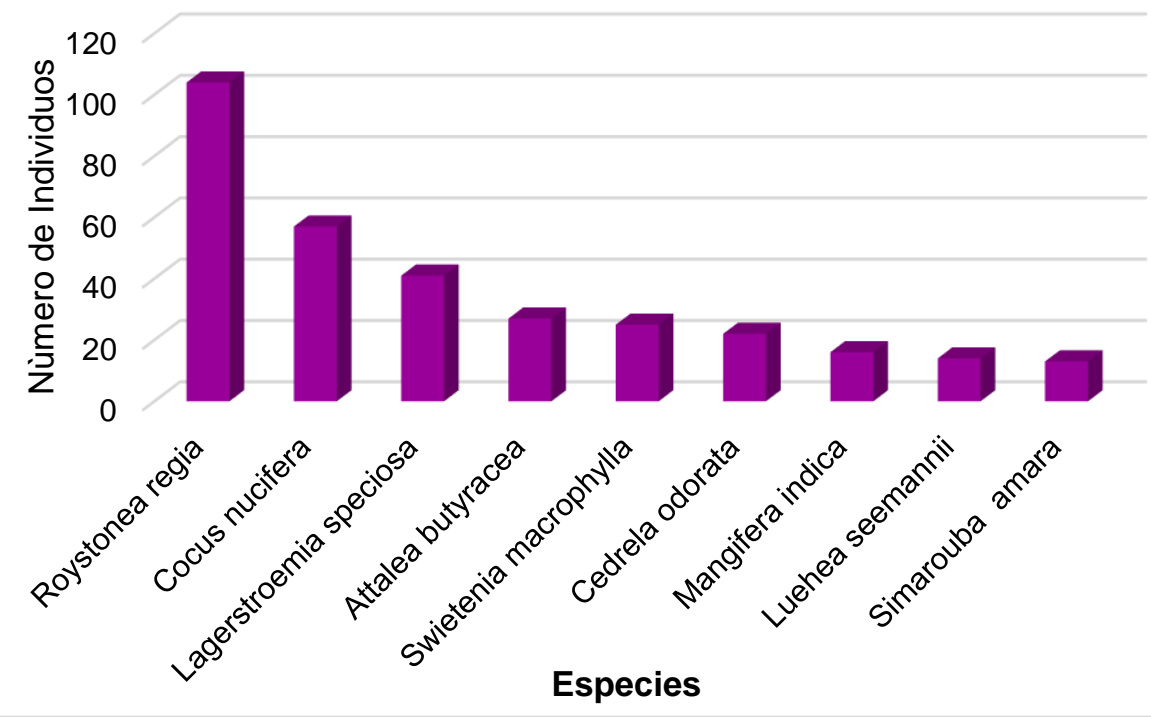

Figura 4. Especies más representativas según el número de individuos en las comunidades de Margarita, Espinar y Davis. Fuente. Elaboración propia. (2019).

\section{B. Riesgo de los árboles}

Los árboles juegan un papel importante en la biodiversidad urbana, ya que proporcionan un hábitat para aves y mamíferos, le proporcionan alimento y protección a la fauna circundante. Estos árboles en el entorno urbano se encuentran día a día sometidos a diversas situaciones, que pueden provocarles distintos peligros como el de ser talados.

Para ello es fundamental conocer la población de árboles presentes en los sitios muestreados, y así identificar esos posibles peligros que influyen en su desarrollo durante el paso de los años.

Los tres sitios muestreados, a partir de la evaluación realizada del área, mostraron que el arbolado en su estado actual tiene poco nivel de riesgo, lo cual está relacionado a que estas son áreas revertidas de la antigua Zona del Canal, son áreas con pocos habitantes y estos son muy respetuosos de no talar los árboles, además son áreas muy bien diseñadas en las que se pensó en conservar siempre los árboles, enfoque que a lo largo de los años ha garantizado la conservación de los mismos por más de cien años. Los árboles cercanos a las residencias son podados eventualmente para evitar riesgos, consideran el derribo de los árboles si se encuentran muertos o presentan un riesgo inminente a desplome. Estas son áreas con muy bajo volumen de tránsito. (ver Figura 5).

En este estudio especies como: Cedrela odorata, Lagerstroemia speciosa, Mangifera indica y Swietenia macrophylla son especies que requieren de mucho mantenimiento sobre todo de poda constante, ya que sus copas son amplias y 
la mayoría de veces tienden a perturbar estructuras importantes en las urbanizaciones estudiadas como las viviendas y demás estructuras locales.

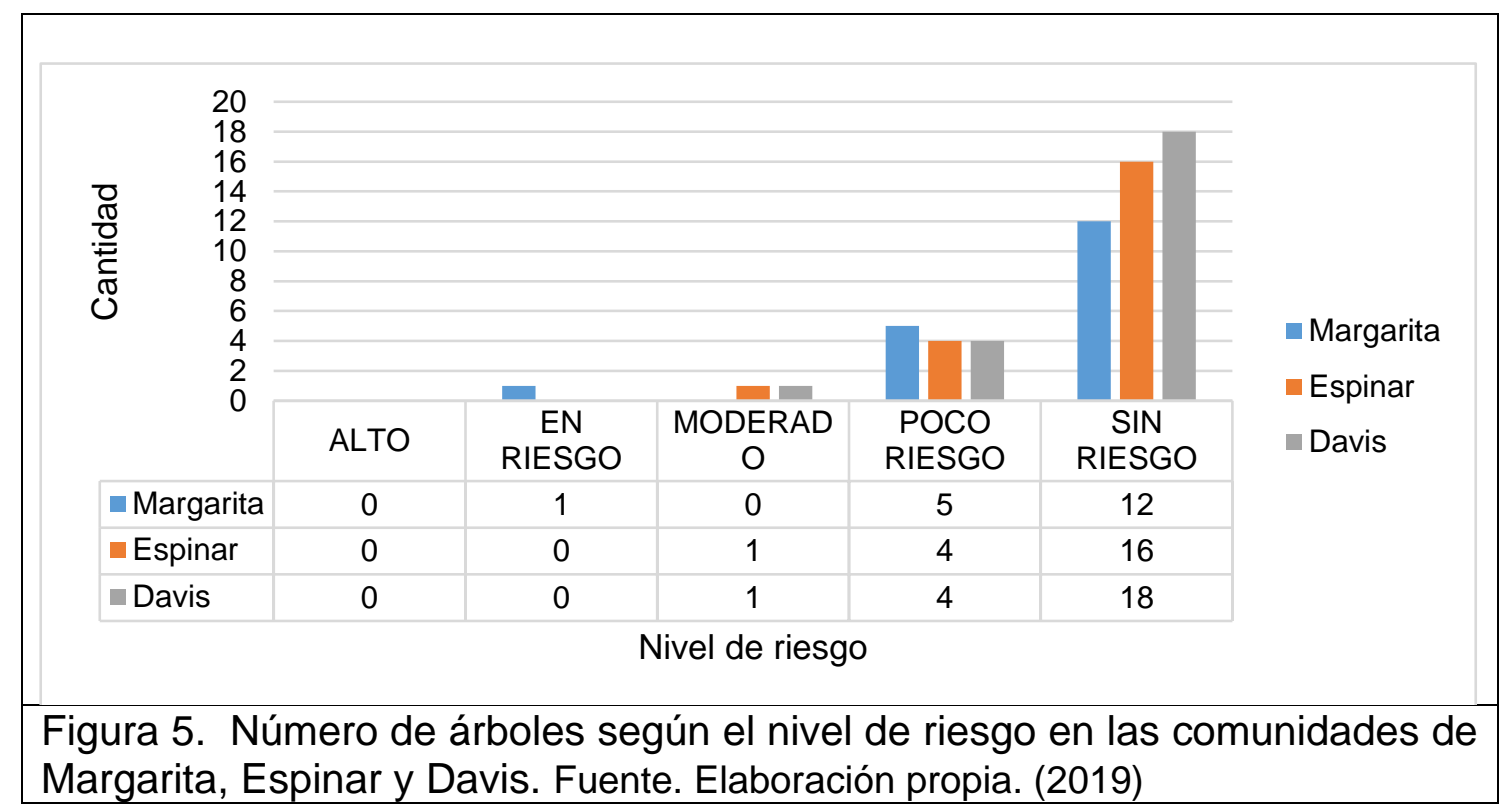

La comunidad considera importante estas especies por su valor e importancia ambiental, social, ecológica, económica entre otros. Estos árboles le dan parte de la belleza escénica a las urbanizaciones creando un ambiente agradable. Por lo que es preciso darles mantenimiento, ya que los mismos tienen una edad avanzada en su mayoría (ver Figura 6 y Tabla 4).

La condición del arbolado urbano en las áreas de estudio se encuentra en buen estado, aunque se encontraron arboles inclinados hacia las casas, otros de gran tamaño, estos pueden dañar las estructuras, algunos producen abundante material orgánico esto es beneficioso para el suelo, pero algunas veces las personas no lo perciben así, a pesar de ello las personas tratan de cuidarlos, considerando que estos árboles tienen un alto valor de uso por parte de la comunidad.

Se considera que la vida útil de los árboles urbanos es corta en comparación a los mismos en estado natural (Román y Scatena, 2011). Lo que indica que se debe considerar la situación fitosanitaria de los árboles urbanos. En este estudio los árboles presentan un buen estado, pero son árboles antiguos, lo que implica que estos pueden tener una menor esperanza de vida sino se le da un mantenimiento adecuado y estricto. Lo que implica que si se quiere seguir contando con espacios verdes, es necesario tener en cuenta la salud y la duración de la vida de los árboles, lo que conducirá a necesidades más rápidas 
de reemplazo, por lo tanto a mayores costos para el manejo y la administración de estos (Soares et al, 2011).

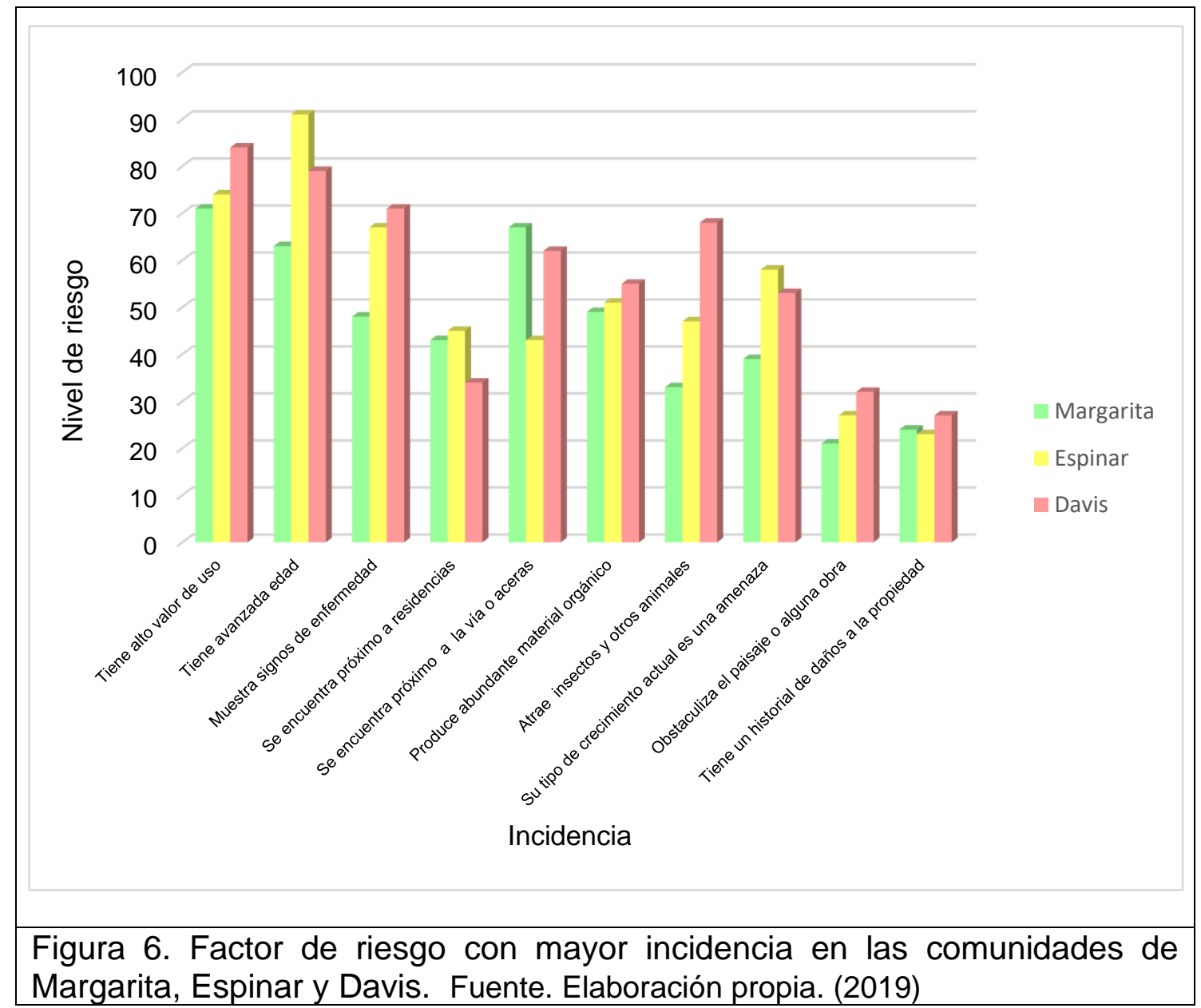

Tabla 4. Factor de riesgo con mayor incidencia combinado

\begin{tabular}{|l|l|l|l|l|l|}
\hline Margarita & \multicolumn{2}{|l|}{ Espinar } & Davis \\
\hline Factor de riesgo & Incidencia & factor de riesgo & Incidencia & $\begin{array}{l}\text { factor de } \\
\text { riesgo }\end{array}$ & incidencia \\
\hline $\begin{array}{l}\text { Tiene alto valor } \\
\text { de uso }\end{array}$ & 71 & $\begin{array}{l}\text { Tiene avanzada } \\
\text { edad }\end{array}$ & 91 & $\begin{array}{l}\text { Tiene alto } \\
\text { valor de uso }\end{array}$ & 84 \\
\hline $\begin{array}{l}\text { Se encuentra } \\
\text { próximo a la vía o } \\
\text { aceras }\end{array}$ & 67 & $\begin{array}{l}\text { Tiene alto valor de } \\
\text { uso }\end{array}$ & 74 & $\begin{array}{l}\text { Tiene } \\
\text { avanzada } \\
\text { edad }\end{array}$ & 79 \\
\hline $\begin{array}{l}\text { Tiene avanzada } \\
\text { edad }\end{array}$ & 63 & $\begin{array}{l}\text { Muestra signos de } \\
\text { enfermedad }\end{array}$ & 67 & $\begin{array}{l}\text { Muestra } \\
\text { signos de } \\
\text { enfermedad }\end{array}$ & 71 \\
\hline $\begin{array}{l}\text { Produce } \\
\text { abundante } \\
\text { material orgánico }\end{array}$ & 49 & $\begin{array}{l}\text { Su tipo de } \\
\text { crecimiento actual } \\
\text { es una amenaza }\end{array}$ & 58 & $\begin{array}{l}\text { Atrae } \\
\text { insectos y } \\
\text { otros } \\
\text { animales }\end{array}$ & 68 \\
\hline $\begin{array}{l}\text { Muestra signos } \\
\text { de enfermedad }\end{array}$ & 48 & $\begin{array}{l}\text { Produce } \\
\text { abundante } \\
\text { material orgánico }\end{array}$ & 51 & $\begin{array}{l}\text { Se } \\
\text { encuentra } \\
\text { próximo a } \\
\text { la vía o } \\
\text { aceras }\end{array}$ & 62 \\
\hline
\end{tabular}




\begin{tabular}{|l|l|l|l|l|l|}
\hline $\begin{array}{l}\text { Se encuentra } \\
\text { próximo a } \\
\text { residencias }\end{array}$ & 43 & $\begin{array}{l}\text { Atrae insectos y } \\
\text { otros animales }\end{array}$ & 47 & $\begin{array}{l}\text { Produce } \\
\text { abundante } \\
\text { material } \\
\text { orgánico }\end{array}$ & 55 \\
\hline $\begin{array}{l}\text { Su tipo de } \\
\text { crecimiento } \\
\text { actual es una } \\
\text { amenaza }\end{array}$ & 39 & $\begin{array}{l}\text { Se encuentra } \\
\text { próximo a } \\
\text { residencias }\end{array}$ & 45 & $\begin{array}{l}\text { Su tipo de } \\
\text { crecimiento } \\
\text { actual es } \\
\text { una } \\
\text { amenaza }\end{array}$ & 53 \\
\hline $\begin{array}{l}\text { Atrae insectos y } \\
\text { otros animales }\end{array}$ & 33 & $\begin{array}{l}\text { Se encuentra } \\
\text { próximo a la vía o } \\
\text { aceras }\end{array}$ & 43 & $\begin{array}{l}\text { Se } \\
\text { encuentra } \\
\text { próximo a } \\
\text { residencias }\end{array}$ & 34 \\
\hline $\begin{array}{l}\text { Tiene un historial } \\
\text { de daños a la } \\
\text { propiedad }\end{array}$ & 24 & $\begin{array}{l}\text { Obstaculiza el } \\
\text { paisaje o alguna } \\
\text { obra }\end{array}$ & 27 & $\begin{array}{l}\text { Obstaculiza } \\
\text { el paisaje o } \\
\text { alguna obra }\end{array}$ & 32 \\
\hline $\begin{array}{l}\text { Obstaculiza el } \\
\text { paisaje o alguna } \\
\text { obra }\end{array}$ & 21 & $\begin{array}{l}\text { Tiene un historial } \\
\text { de daños a la } \\
\text { propiedad }\end{array}$ & 23 & $\begin{array}{l}\text { Tiene un } \\
\text { historial de } \\
\text { daños a la } \\
\text { propiedad }\end{array}$ & 27 \\
\hline
\end{tabular}

Fuente. Elaboración propia. (2019).

\section{B. Usos Etnobotánicos}

Las especies registradas en su mayoría, presentan más de dos usos etnobotánicos lo que implica que tienen varios usos. Se identificaron 7 categorías etnobotánicas: Alimento, ornamental, medicinal, madera, artesanal, industrial y tradiciones (ver Tabla 5). Especies como Anacardium occidentale y Ochroma pyramidale registraron 6 usos. Además se reportaron 28 especies con uso ornamental, 25 especies con uso maderable y 23 con uso medicinal. A pesar de que estas son urbanizaciones, las personas hacen uso de las plantas circundantes (ver Tabla 6), hay una estrecha relación de uso con los recursos vegetales del entorno en estas áreas.

Tabla 5. Usos etnobotánicos y número de especies 


\begin{tabular}{|l|l|}
\hline Usos & Número de especies \\
\hline Ornamental & 28 \\
\hline Madera & 25 \\
\hline Medicinal & 23 \\
\hline Ornamental & 21 \\
\hline Artesanal & 15 \\
\hline Industrial & 5 \\
\hline Tradiciones & 5 \\
\hline Fuente. Elaboración propia. (2019) \\
\hline
\end{tabular}

Tabla 6. 


\begin{tabular}{|c|c|c|c|c|c|c|c|c|c|}
\hline \multicolumn{10}{|c|}{ Usos etnobotánicos } \\
\hline & & \begin{tabular}{|l|} 
Alimento \\
\end{tabular} & Ornamental & Medicinal & Madera & Artesanal & \begin{tabular}{|l|} 
Industrial \\
\end{tabular} & Tradiciones & Total \\
\hline $\begin{array}{l}\text { Nombre } \\
\text { Común }\end{array}$ & Nombre cientifico & & & & & & & & \\
\hline Espavé & $\begin{array}{l}\text { Anacardium } \\
\text { excelsum }\end{array}$ & 1 & 1 & 1 & 1 & 1 & & 1 & 6 \\
\hline Balso & $\begin{array}{l}\text { Ochroma } \\
\text { pyramidale }\end{array}$ & 1 & 1 & 1 & 1 & 1 & & 1 & 6 \\
\hline Indio encuero & Bursera simaruba & & 1 & 1 & 1 & 1 & & 1 & 5 \\
\hline Marañón & $\begin{array}{l}\text { Anacardium } \\
\text { occidentale }\end{array}$ & 1 & 1 & & 1 & & 1 & & 4 \\
\hline Palma real & Attalea butyracea & 1 & & 1 & & 1 & & 1 & 4 \\
\hline Bambú & Bambusa vulgaris & 1 & 1 & & 1 & 1 & & & 4 \\
\hline Cedro amargo & Cedrela odorata & & 1 & 1 & 1 & 1 & & & 4 \\
\hline Caimito & $\begin{array}{l}\text { Chrysophyllum } \\
\text { cainito }\end{array}$ & 1 & 1 & 1 & 1 & & & & 4 \\
\hline Palma de coco & Cocus nucifera & 1 & 1 & 1 & 1 & & & & 4 \\
\hline Guácimo & Guazuma ulmifolia & 1 & & 1 & 1 & 1 & & & 4 \\
\hline Tronador & Hura crepitans & 1 & 1 & 1 & 1 & & & & 4 \\
\hline Cedro espino & Pachira quinata & & 1 & 1 & 1 & 1 & & & 4 \\
\hline Caoba & $\begin{array}{l}\text { Swietenia } \\
\text { macrophylla }\end{array}$ & & 1 & & 1 & 1 & 1 & & 4 \\
\hline Guayacán & $\begin{array}{l}\text { Tabebuia } \\
\text { guayacan }\end{array}$ & & 1 & 1 & 1 & 1 & & & 4 \\
\hline Laurel & Cordia alliadora & & 1 & & 1 & 1 & & & 3 \\
\hline Biyuyo & Cordia dentata & 1 & & 1 & 1 & & & & 3 \\
\hline Corotú & $\begin{array}{l}\text { Enterolobium } \\
\text { cyclocarpum }\end{array}$ & & 1 & & 1 & & 1 & & 3 \\
\hline Higuerón & Ficus insipida & 1 & 1 & & 1 & & & & 3 \\
\hline Guaba & Inga spectabilis & 1 & 1 & & 1 & & & & 3 \\
\hline Mango & Mangifera indica & 1 & 1 & & 1 & & & & 3 \\
\hline Dos caras & Miconia argentea & & & & 1 & 1 & & 1 & 3 \\
\hline Noni & $\begin{array}{l}\text { Morinda } \\
\text { panamensis }\end{array}$ & 1 & 1 & 1 & & & & & 3 \\
\hline Cheflera & $\begin{array}{l}\text { Schefflera } \\
\text { morototoni }\end{array}$ & & 1 & & 1 & 1 & & & 3 \\
\hline Aceituno & Simarouba amara & 1 & & 1 & & & 1 & & 3 \\
\hline Ciruela & Spondias purpurea & 1 & 1 & 1 & & & & & \\
\hline Almendro & Terminalia catappa & 1 & 1 & 1 & & & & & 3 \\
\hline Guanábana & Anona muricata & 1 & & 1 & & & & & 2 \\
\hline Bala de cañón & $\begin{array}{l}\text { Couroupita } \\
\text { guianensis }\end{array}$ & & & 1 & 1 & & & & 2 \\
\hline Membrillo & Gustavia superba & 1 & & 1 & & & & & 2 \\
\hline Nazareno & Jacaranda copaia & & 1 & & 1 & & & & 2 \\
\hline $\begin{array}{l}\text { Guácimo } \\
\text { colorado }\end{array}$ & $\begin{array}{l}\text { Couroupita } \\
\text { guianensis }\end{array}$ & & & & 1 & 1 & & & 2 \\
\hline Moringa & Moringa oleifera & & & 1 & & & 1 & & 2 \\
\hline Coralillo & Ormosia coccinea & & 1 & & & 1 & & & 2 \\
\hline Aguacate & Persea americana & 1 & & 1 & & & & & 2 \\
\hline Jobo & Spondias mombin & 1 & & 1 & & & & & 2 \\
\hline Roble & Tabebuia rosea & & 1 & & 1 & & & & 2 \\
\hline Poro poro & $\begin{array}{l}\text { Cochlospermum } \\
\text { vitifolium }\end{array}$ & & & 1 & & & & & 1 \\
\hline Astromelia & $\begin{array}{l}\text { Lagerstromeia } \\
\text { speciosa }\end{array}$ & & 1 & & & & & & 1 \\
\hline Palma cubana & Roystonea regia & & 1 & & & & & & 1 \\
\hline $\begin{array}{l}\text { Marañón } \\
\text { curasao }\end{array}$ & $\begin{array}{l}\text { Syzygium } \\
\text { malaccense }\end{array}$ & & 1 & & & & & & 1 \\
\hline Zyzygium & zyzygium sp. & & 1 & & & & & & 1 \\
\hline Caria & Carya sp. & & & & & & & & \\
\hline TOTAL & & 21 & 28 & 23 & 25 & 15 & 5 & 5 & 122 \\
\hline
\end{tabular}

Fuente. Elaboración propia. (2019) 


\section{Especies De Interes}

Los bosques de los sitios de estudio mostraron un alto interés de conservación pues $35 \%$ de las especies presentan algún grado de interés para su conservación, según los organismos evaluadores de la conservación nacional y mundial (ANAM (2020), CITES (2020), UICN (2020)).

Particularmente, se identificaron 3 especies en estado de conservación preocupante, entre ellas: Cedrela odorata, Swietenia macrophylla y Tabebuia guayacan, especies nativas en estado de vulnerabilidad; $y$ otras especies como Cochlospermum vitifolium, Cordia dentata, Couropita guianensis, Guazuma ulmifolia, Gustavia superba, Miconia argentea entre otras, se consideran de preocupación menor.

\begin{tabular}{|l|c|c|c|}
\hline \multicolumn{4}{|l|}{ Tabla 7. Estado de Conservación de las especies observadas. } \\
\hline Especie & CITES & ANAM & UICN \\
\hline Carya sp. & & & LC \\
\hline Cedrela odorata & III & & VU \\
\hline Cochlospermum vitifolium & & & LC \\
\hline Cocus nucifera & & & NT \\
\hline Cordia dentata & & & LC \\
\hline Couroupita guianensis & & & LC \\
\hline Enterolobium cyclocarpum & & & LC \\
\hline Ficus insipida & & & LC \\
\hline Guazuma ulmifolia & & & LC \\
\hline Gustavia superba & & & LC \\
\hline Inga spectabilis & & & LC \\
\hline Mangifera indica & & & DD \\
\hline Miconia argentea & & & LC \\
\hline Morinda panamensis & & & LC \\
\hline Persea americana & & & LC \\
\hline Schefflera morototoni & & & LC \\
\hline Simarouba amara & & & LC \\
\hline Spondias mombin & & & LC \\
\hline Spondias purpurea & II & CR & VU \\
\hline Swietenia macrophylla & & & LC \\
\hline Syzygium malaccense & & VU & VU \\
\hline Tabebuia guayacan & & & VUach \\
\hline $\begin{array}{l}\text { Nota: ANAM: Autoridad Nacional del Ambiente. (2020), UICN: Datos Insuficientes } \\
\text { (DD), Preocupación Menor (LC), Vulnerable (VU), En Peligro Crítico (CR). } \\
\text { (versión CITES: APENDICE II (Casi Amenazada), APENDICE III (Requiere } \\
\text { Protección). (versión 2020.1). Fuente. Elaboración propia. (2019). }\end{array}$ \\
\hline
\end{tabular}




\section{Origen De Las Especies Vegetales}

Con base en las observaciones en campo y la revisión de la literatura (Baptiste, et al., 2010), se observó que la distribución de los diferentes taxones en los tres sitios de estudio se agrupaba en las siguientes categorías: Nativa, Introducida, cultivada y naturalizada; de las cuales, la mayoría de estas corresponden a especies nativas lo que le da un valor adicional para la conservación de estos bosques urbanos pues ellos mantienen la genética de la ecología de las áreas. (Tabla 8).

\begin{tabular}{|c|c|}
\hline Especies & Origen \\
\hline $\begin{array}{l}\text { Anacardium excelsum, Annona muricata, } \\
\text { Attalea butyracea, Bursera simaruba, Cedrela } \\
\text { odorata, Cochlospermum vitifolium, Cordia } \\
\text { alliadora, Cordia dentata, Couroupita } \\
\text { guianensis, Enterolobium cyclocarpum, Ficus } \\
\text { insípida, Guazuma ulmifolia, Gustavia } \\
\text { superba, Hura crepitans, Inga spectabilis, } \\
\text { Jacaranda copaia, Luehea seemannii, } \\
\text { Miconia argéntea, Morinda panamensis, } \\
\text { Ochroma pyramidale, Ormosia coccinea, } \\
\text { Pachira quinata, Schefflera morototoni, } \\
\text { Simarouba amara, Spondias mombin, } \\
\text { Tabebuia guayacan, Tabebuia rosea }\end{array}$ & Nativa \\
\hline Carya sp. & Introducida \\
\hline Chrysophyllum cainito & Cultivada \\
\hline Persea americana & Cultivada \\
\hline Spondias purpurea & Cultivada \\
\hline Swietenia macrophylla & Cultivada \\
\hline Anacardium occidentale & Introducida, cultivada, naturalizada \\
\hline Cocos nucifera & Introducida, cultivada, naturalizada \\
\hline Mangifera indica & Introducida, cultivada, naturalizada \\
\hline Bambusa vulgaris & $\begin{array}{l}\text { Introducida, cultivada } \\
\end{array}$ \\
\hline Lagerstroemia speciosa & Introducida, cultivada \\
\hline Moringa oleifera & Introducida, cultivada \\
\hline Roystonea regia & Introducida, cultivada \\
\hline Syzygium malaccense & Introducida, cultivada \\
\hline Syzygium sp. & Introducida, cultivada \\
\hline Terminalia catappa & Introducida, cultivada \\
\hline Fuente. Elaboración propia. (2019). & \\
\hline
\end{tabular}




\section{Conclusiones}

La realidad de los bosques urbanos es que se encuentran amenazados como cualquier bosque de origen natural y con ellos toda la diversidad que en ellos se desarrolla. Los bosques de Margarita, Davis y Espinar no escapan de esta realidad y aunque el arbolado es de avanzada edad carece de poco riesgo fitosanitario y mecánico es necesario el mantenimiento de los mismos, ya que albergan especies en estado vulnerable, las cuales son de especial interés para la conservación, estas con otras especies nativas y naturalizadas, permiten espacios de conectividad entre espacios naturales y urbanos, permitiendo la creación de corredores biológicos que permiten la presencia de la fauna asociada a estos bosques urbanos, que implica anidación y refugio para estas especies.

La participación ciudadana es necesaria para la conservación de los bosques urbanos, ya que el crecimiento de la urbanizaciones es el principal causante del cambio que se genera en el componente arbóreo de las áreas en estudio, los bosques urbanos al estar expuesto son muy vulnerables a perderse. Son las comunidades organizadas las garantes de cuidar y proteger estos bosques que día a día están siendo más valorados por su beneficio ambiental y ecológico.

El ser humano siempre ha tenido una estrecha relación con las plantas que le rodean, pues son un recurso que le brindan medicina, madera, alimento, entre otros beneficios, que son necesarios para la vida sana del mismo.

Este estudio aporta información valiosa que ayudará a que se elaboren programas de manejo, que permitan el mantenimiento adecuado de las especies vegetales de los bosques urbanos del área de estudio y así se puedan desarrollar estrategias que ayuden a la conservación de los mismos. 


\section{Referencias Bibliográficas}

ANAM (2020). Autoridad Nacional del Ambiente. Recuperado de https://www.miambiente.gob.pa/?s=lista+de+especies+protegidas. Fecha: 5 de mayo 2020.

Balvanera, P. (2012). Los servicios ecosistémicos que ofrecen los bosques tropicales. Ecositesmas 21: 136-147.

Baptiste M.P., Castaño N., Cárdenas D., Gutiérrez F. P., Gil D.L. y Lasso C.A. (eds). 2010. Análisis de riesgo y propuesta de categorización de especies introducidas para Colombia. Instituto de Investigación de Recursos Biológicos Alexander von Humboldt. Bogotá, D. C., Colombia. 200 p.

CITES. (2020). Convención sobre el Comercio Internacional de Especies Amenazadas de Fauna y Flora Silvestres. Recuperado de: https://cites.org/esp. Fecha; 12 de septiembre de 2020.

Diaz, Vásquez, J. y Ballesteros, A. (2012). Bosques urbanos para enfriar las ciudades. Academia Mexicana de Ciencias, Vol 63 (4): 36-41.

Farnum, F. y Murillo, V. (2014). Árboles y arbustos de los predios del Centro Regional Universitario de Colón. Primera Edición. Universidad de Panamá. 124 pp.

FAO. 2016. Directrices para la silvicultura urbana y periurbana, por F. Salbitano, S. Borelli, M. Conigliaro e Y. Chen. Estudio FAO: Montes N.ำ178. Roma.

Ibrahim, M. Chacón, m., Cuartas, C., Naranjo, J., Ponce, C., Vega, P., Casasola, F. y Rojas, J. (2007). Almacenamiento de Carbono en el suelo y la biomasa arbórea en sistemas de usos de la tierra en paisajes ganaderos de Colombia, Costa Rica y Nicaragua. Agroforestería en las Américas 45 (1): 27-36.

Koeser, A., Hauer, R., Miesbauer, J. y Peterson, W. (2016). Municipal tree risk in the United States: Findings from a comprehensive survey of urban forest management. Arboriculture Journal, 38(4), 218229. DOI: https://doi.org/10.1080/03071375.201 6.1221178.

Lawrence, A., De Vreese, R., Johnston, M., Konijnendijk van den Bosch, C.C. y Sanesi, G. (2013). Urban forest governance: towards a framework for comparing approaches. Urban Forestry and Urban Greening, 12: 464473. 
O'Brien, J. (2003). Introduction. En, Urban Tree Risk Management: A Community Guide to Program Design and Implementation. Saint Paul, EE. UU: USDA Forest Service. Recuperado de http://www. na.fs.fed.us/spfo/pubs/uf/ utrmm/urban_tree_risk_mgmnt.pdf. https://doi.org/10.1201/b16997-2.

ONU-Hábitat. 2015. International guidelines on urban and territorial planning.

Nairobi, Programa de las Naciones Unidas sobre los Asentamientos Humanos. (ONU-Hábitat)

Roman, Lara A., Scatena, F.N. (2011). Street tree survival rates: Metanalysis of previous studies and application to a field survey in Philadelphia, PA, USA. Urban Forestry \& Urban Greening 10: 269-274.

Soares, A. L., Regoa, F. C., McPherson, E.G., Simpson, J.R., Pepper, P.J., Xiao, Q. (2011) Benefits and costs of street trees in Lisbon, Portugal. Urban Forestry \& Urban 10: 69-78.

Tomao, A., Secondi, A., Corona, P., Giuliarelli, D., Quantrini, V. y Agrimi, M. (2015). Can composite indices explain multidimensionality of tree risk assessment? A case study in an historical monumental complex. Urban Forest \& Urban Greening, 14(3), 456-465. DOI: https://doi.org/10.1016/j. ufug.2015.04.009.

Trzyna, T. (2014). Urban protected areas: profiles and best practice guidelines. Best Practice Protected Area Guidelines Series No. 22, Gland, S witzerland: IUCN. xiv +110pp.

UICN (2020). LA LISTA ROJA DE ESPECIES AMENAZADAS DE LA UICN. Recuperada de https://www.iucn.org/resources/conservation-tools/iucnred-list-threatened-species Fecha; 6 de julio de 2020. 